\title{
Estimaciones de atresia mayor en la temporada reproductiva 1999-2000 en la sardina monterrey (Sardinops sagax) en Bahía Magdalena, México
}

\author{
Major atresia assessment in the Pacific sardine (Sardinops sagax) in the 1999-2000 \\ reproductive season, off Bahia Magdalena, Mexico
J. René Torres-Villegas ${ }^{1,2}$, Rosa Isabel Ochoa-Báez ${ }^{1,2,3}$, Liduvina Perezgómez ${ }^{1}$ y Gustavo García-Melgar ${ }^{1}$ \\ ${ }^{1}$ Laboratorio de Morfofisiología. CICIMAR-IPN. Playa Palo de Santa Rita s/n. 23090. La Paz Baja California Sur, México \\ ${ }^{2}$ Becario de la Comisión de Operación y Fomento de las Actividades Académicas. \\ ${ }^{3}$ Becario del Sistema de Estímulos al Desempeño de la Investigación del IPN \\ jvillega@ipn.mx
}

\begin{abstract}
The gonadosomatic index (GSI) and the gonadic relative index (GRI) were used for monthly monitoring of Pacific sardine maturity in Bahia Magdalena, Mexico, for the reproductive season of 1999-2000. These indices showed an increase in gonadic growth in January and March, and a decline in February. Histological analysis of ovaries showed a short spawning season. Juvenile fish were recorded in December. Adults appeared until January. In February, high incidence of atresia in oocytes at the cortical alveolli stage was detected. Later, atresia occurred in advanced vitellogenic oocytes. The active female frequency ranged from 11 to $55 \%$ in the spawning season of $1999-2000$. Atresia on isolated oocyte frequency was $9 \%$ in January 2000, at the beginning of the spawning season. The incidence of major atresia started in February, and reached 54\% in March, indicating the end of the spawning season. These results were compared with time series from 1980-1990 on Pacific sardine reproduction in Bahia Magdalena. Evidence indicates that the Pacific sardine spawning season was reduced to two months, and that the active female frequency was low. Environmental influence on ovary reabsorption and skip reproduction season is discussed.
\end{abstract}

Key words: Pacific sardine, reproduction, active female, atresia
Resumen.- Durante la temporada de pesca 1999-2000 en Bahía Magdalena, México, se hizo un seguimiento de la reproducción de la sardina monterrey por medio de los índices gonadosomático (IGS) y gonádico relativo (IGR). En diciembre se registraron solo sardinas juveniles y los adultos se encontraron hasta enero. Se observó una variación atípica con respecto a años anteriores; un incremento del peso de la gónada en enero, declinación en febrero y una recuperación nuevamente en marzo. El análisis histológico de los ovarios mostró que la actividad gametogénica se inició en enero y terminó en marzo. Desde el inicio de la temporada reproductiva en enero de 2000 se registró una alta incidencia de atresia en oocitos en estadio de alveolo cortical (9\%). En marzo, cuando normalmente se presenta el máximo de puesta se registró atresia también en oocitos en vitelogénesis avanzada y atresia mayor con una frecuencia de 90\%. Lo anterior indicó una interrupción de la temporada reproductiva evidenciándose por una frecuencia mínima de hembras reproductivamente activas. Se discute la influencia del ambiente en la reabsorción ovárica y en la interrupción de la reproducción de la sardina monterrey.

Palabras clave: Sardina monterrey, reproducción, hembras activas, atresia

\section{Introducción}

La sardina monterrey Sardinops sagax (Jenyns, 1842), es una especie iterópara con desarrollo de los oocitos intraováricos asincrónico (Torres-Villegas et al. 1985). La distribución de diámetros de los oocitos intraováricos es polimodal. En el ovario de la sardina pueden identificarse oocitos en todos los estados de desarrollo, desde oogonias hasta estadios postovulatorios de 0,1 , y 2 días, después de la puesta (Torres-Villegas 1986). En numerosas especies de peces, el epitelio germinal se mantiene activo durante la temporada de reproducción, lo que indica la producción continua de nuevas células sexuales (Grier 
2000). Con frecuencia una pequeña parte de oocitos detiene su desarrollo y es reabsorbida, manifestándose por el proceso denominado atresia folicular (Byskov 1978). En algunas especies de peces la alta incidencia de atresia folicular determina que las estimaciones de la fecundidad parcial sean corregidas con un índice de atresia (Hunter et al. 1985, Priede et al. 1994). Livingstone et al. (1997) utilizó la tasa de atresia como un índice de corrección para las proyecciones del reclutamiento en Macruronus novaezelandiae. Lo mismo ocurrió con el manejo del stock de Hoplostethus atlanticus en el Pacífico Suroriental (Bell et al. 1992).

En S. sagax, la incidencia de oocitos aislados con atresia, a lo largo de la temporada de puesta, se considera normal cuando se presentan alrededor del $2 \%$. Cuando esta frecuencia alcanza el $5 \%$, es indicador del final del máximo de puesta. La atresia mayor consiste en la reabsorción generalizada de oocitos intraováricos (mas del 50\% de los oocitos afectados por atresia). De acuerdo con los criterios de Hunter \& Macewicz (1985), al llegar a este nivel de reabsorción se asume la reducción de la probabilidad de que esas hembras alcancen el desove nuevamente. Este estadio se presenta al final de la temporada reproductiva e indica el fin de la producción de oocitos (TorresVillegas et al. 1985, Torres-Villegas 1997).

El proceso de atresia mayor en poblaciones silvestres de peces marinos es un tema poco estudiado (Hunter \& Macewicz 2003). Existe evidencia de variaciones en la temporada de puesta de $S$. sagax en Chile, descritas por Claramunt \& Roa (2001). TorresVillegas et al. (1992) encuentra en Bahía Magdalena, México, variaciones de la reproducción en la misma especie. Las variaciones de la temporada reproductiva han sido poco mencionadas en relación con la reabsorción de oocitos intraováricos.

La atresia mayor puede presentarse desde los primeros estados de madurez del oocito y del folículo hasta justo antes del desove. Las causas de la atresia mayor antes de la madurez del oocito fueron estudiadas por Fedorov (1971) y Burton \& Idler (1984) quienes asocian la reabsorción de oocitos intraováricos con temperaturas bajas en las masas de agua donde están las sardinas. Los mismos autores discuten sobre el efecto de una pobre condición nutricional o inanición al final de la puesta. Esta relación ha sido documentada en experimentos de laboratorio por diversos autores (Scott 1962, Bagenal 1969, Wooton 1973, Hislop et al. 1978,
Kjesbu et al. 1991, Rjinsdrop 1990, Maddock \& Burton 1994). Lam et al. (1978) analizaron los efectos del hacinamiento en la retención de oocitos maduros en peces juveniles, así como cambios en la proporción sexual (Swingle 1956, Trippel \& Harvey 1990). La contaminación del agua también puede causar interrupciones de la reproducción (Beamish et al. 1975, McFarland \& Franzin 1978). En especies tales como Gadus morhua hay reportes de suspensión de la reproducción, a pesar de haber alcanzado la madurez (Messiatzeva 1932, Shirokova 1969, Burton et al. 1997, Rideout et al. 2000).

Durante la temporada de pesca 1999-2000, en Bahía Magdalena, se registró una alta incidencia de sardina monterrey por debajo de la talla de primera madurez $(<$ $150 \mathrm{~mm}$ ), por lo que se desarrolló una investigación histológica en los ovarios de esas sardinas con el objetivo de conocer el estado reproductivo de la población con alta incidencia de juveniles en la temporada de puesta.

\section{Material y métodos}

Se tomaron muestras mensuales entre diciembre de 1999 y noviembre de 2000 de $S$. sagax a bordo de la flota comercial sardinera de Bahía Magdalena B. C. S. México (Fig. 1). Por cada lance de pesca muestreado se tomaron entre 60 y 100 sardinas antes de embarcar la captura. Los peces fueron tomados al azar; el tamaño de muestra varió dependiendo de las facilidades para el muestreo a bordo y de las condiciones del tiempo. Inmediatamente después de la recolecta, a cada uno de los peces muestreados se les hizo un corte a lo largo de la línea media ventral y por ambos lados de la pared del cuerpo. Las sardinas fueron fijadas completas en formol al $10 \%$ en solución reguladora de fosfatos a $\mathrm{pH}$ 7,0 . Todos los ejemplares fueron muestreados vivos y el tiempo máximo transcurrido entre la muerte y la fijación fue de aproximadamente 30 minutos. Debido a que el máximo de puesta de la sardina monterrey, en el área de estudio, se presenta entre diciembre y abril (Torres-Villegas et al. 1995), se incrementó el muestreo tanto como lo permitieron las condiciones del tiempo en este período. La Tabla 1 presenta los detalles del muestreo empleado para este estudio.

Para cada ejemplar se registró la longitud patrón, el peso total, y el peso de las gónadas (Hunter et al. 1985, Torres-Villegas et al. 1985). 


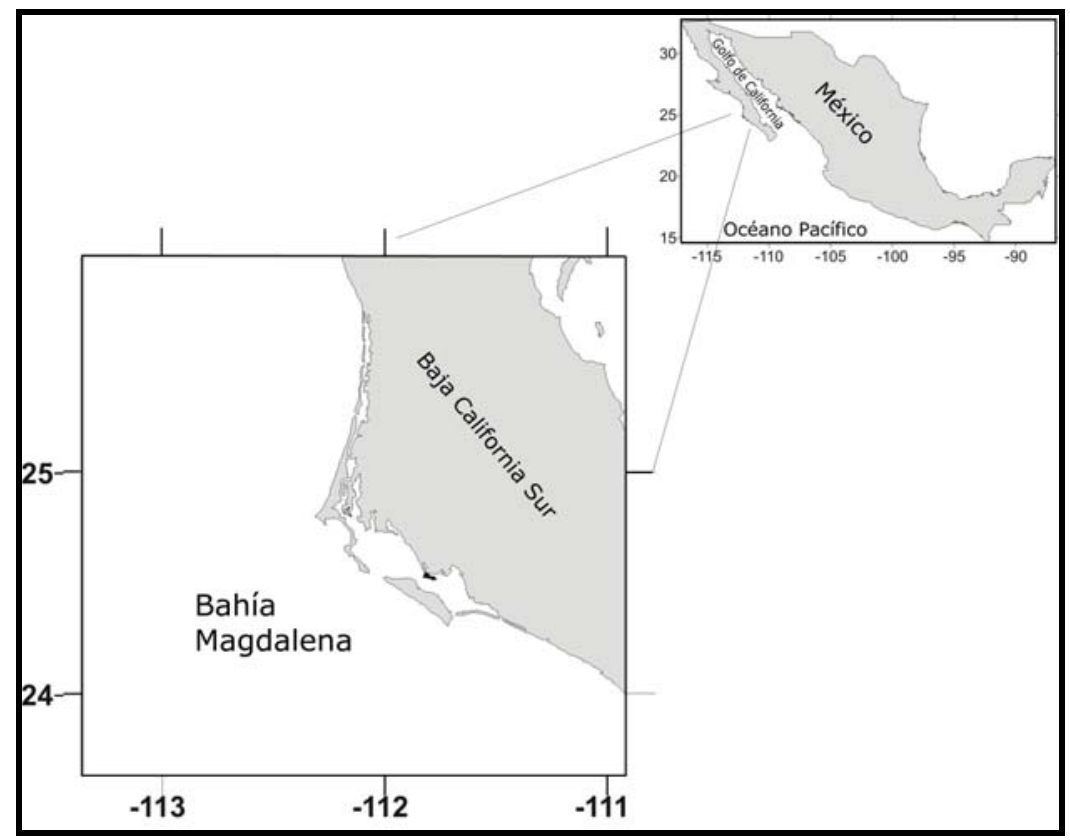

Figura 1

\section{Área de influencia de la pesquería de S. sagax en la costa occidental de Baja California Sur}

S. sagax exploitation area along the west coast of the South Baja California

A partir de los datos morfométricos se obtuvo la distribución de tallas y se hizo la regresión entre el peso libre de las gónadas y la longitud patrón con una transformación logarítmica para cada mes. Se calcularon los índices gonadosomático (Macer 1974)

$$
G S I=\left(\frac{w_{g}}{w_{t}-w_{g}}\right) 100
$$

gonádico relativo (Erickson et al. 1985)

$$
R G I=\left(\frac{\ln w_{g}}{\beta\left(w_{t}-w_{g}\right)}\right)-\alpha,
$$

y el factor de condición (Tesch 1971)

$$
F C=\left(\frac{w_{t-o b s}}{w_{t-c a l c}}\right),
$$

donde

$$
\begin{aligned}
& w_{t}=\text { peso total } \\
& w_{g}=\text { peso de las gónadas }
\end{aligned}
$$

$\alpha \mathrm{y} \beta$ son los coeficientes de la regresión entre el peso libre de gónadas y la longitud patrón.

El ciclo de madurez sexual fue seguido durante el año con estos índices. Los datos y tamaño de muestra también se presentan en la Tabla 1.

Las gónadas de todos los especimenes muestreados entre diciembre de 1999 y abril de 2000 fueron analizadas por métodos histológicos. De un total de 2579 sardinas muestreadas, se analizaron con histología 1967. El análisis histológico se realizó en alícuotas de $0,5 \mathrm{~cm}^{3}$ de la región media de las gónadas. Entre mayo y noviembre, aunque no se tiene actividad reproductiva de la especie, se tomaron al azar sub-muestras de 20 
Tabla 1

Resumen del muestreo mensual de sardina monterrey (S. sagax) en Bahía Magdalena para la temporada reproductiva 1999-2000

Pacific sardine (S. sagax) montly sampling resume in reproductive season 1999-2000 in Bahia Magdalena

\begin{tabular}{|c|c|c|c|c|c|c|c|}
\hline Fecha & $\begin{array}{c}\text { Lances de } \\
\text { pesca } \\
\text { muestreados }\end{array}$ & $\begin{array}{l}\text { Número de } \\
\text { especimenes }\end{array}$ & $\begin{array}{c}\text { Muestras } \\
\text { procesadas por } \\
\text { histología }\end{array}$ & $\begin{array}{c}\text { Número de } \\
\text { hembras }\end{array}$ & $\begin{array}{c}\text { Especimenes } \\
\text { descartados }\end{array}$ & Causa & Localidad \\
\hline Dic-99 & 4 & 246 & 60 & 162 & 186 & Gónadas < 0,1g & Interior/exterior \\
\hline Ene-00 & 4 & 257 & 257 & 195 & 0 & & Interior \\
\hline Feb-00 & 1 & 100 & 100 & 45 & 0 & & Interior \\
\hline Abr-00 & 10 & 616 & 616 & 352 & 0 & & Interior/exterior \\
\hline May-00 & 2 & 180 & 60 & 59 & 120 & Gónadas $<0,1 \mathrm{~g}$ & Interior/exterior \\
\hline Jun-00 & 1 & 60 & 20 & 32 & 40 & Gónadas < 0,1g & Exterior \\
\hline Jul-00 & 2 & 120 & 20 & 61 & 100 & Gónadas < 0,1g & Interior \\
\hline Ago-00 & 1 & 60 & 20 & 27 & 40 & Gónadas $<0,1 \mathrm{~g}$ & Interior \\
\hline Sep-00 & 2 & 120 & 40 & 59 & 80 & Gónadas $<0,1 \mathrm{~g}$ & Interior \\
\hline Oct-00 & 1 & 60 & 20 & 32 & 40 & Gónadas $<0,1 \mathrm{~g}$ & Interior \\
\hline Nov-00 & 1 & 60 & 20 & 31 & 40 & Gónadas < 0,1g & Interior \\
\hline Totales & 40 & 2639 & 1967 & 1561 & 652 & & \\
\hline
\end{tabular}

Nota: Interior, Exterior se refiere a pescas realizadas dentro o fuera de Bahía Magdalena

sardinas, las cuales se analizaron por histología. Este análisis se hizo para calibrar el monitoreo mensual con los índices de peso GSI y GRI. Las alícuotas fueron incluidas en parafina y se obtuvieron cortes entre 3-5 $\mu \mathrm{m}$ de espesor. Los cortes fueron coloreados alternativamente con las técnicas Hematoxilina Eosina, Tricrómica de Mallory o Van Gieson (Martoja \& Martoja-Pierson 1970, Lowe 1997), dependiendo de la disponibilidad en el laboratorio; los resultados de estas técnicas son equivalentes debido a que se les considera como métodos generales de diagnóstico. La terminología empleada para describir la madurez del oocito, así como las fracciones de la población a través de su estado reproductivo se tomaron de Hunter et al. (1992). Los criterios morfológicos usados en el diagnóstico histológico en los estadios preovulatorios fueron tomados de Wallace \& Selman (1981), OchoaBáez (1998), y Torres-Villegas et al. (2000). Los folículos postovulatorios fueron identificados siguiendo los criterios de Hunter \& Macewicz (1980), Alarcón et al. (1984), y Torres-Villegas (1997). Los estados atrésicos fueron identificados con los criterios de Lambert (1970) y Hunter \& Macewicz (1985). La frecuencia de hembras activas (Hunter et al. 1992) fue usada como un indicador de la intensidad de la puesta a lo largo de la temporada.

La frecuencia de atresia fue calculada con las hembras que mostraron oocitos con algún estado de reabsorción. El estadio de atresia mayor fue diferenciado en aquellos ovarios donde la reabsorción es un proceso generalizado, es decir, cuando afecta aproximadamente al 50\% de los oocitos intraováricos. En esta etapa, por lo general, se presentan diferentes estados de atresia por campo del microscopio.

Los resultados del análisis histológico se presentan como frecuencias por estado de madurez. Todos los cálculos fueron hechos con el paquete MINITAB (rel. 13.32).

\section{Resultados}

En diciembre, cuando se inició la temporada de puesta, se encontró una alta incidencia de sardinas juveniles con tallas menores a $150 \mathrm{~mm}(\bar{X}=138 \mathrm{~mm})$. Los adultos $(\bar{X}=185 \mathrm{~mm})$ se presentaron en enero; sin embargo, la proporción de juveniles se mantuvo alta 


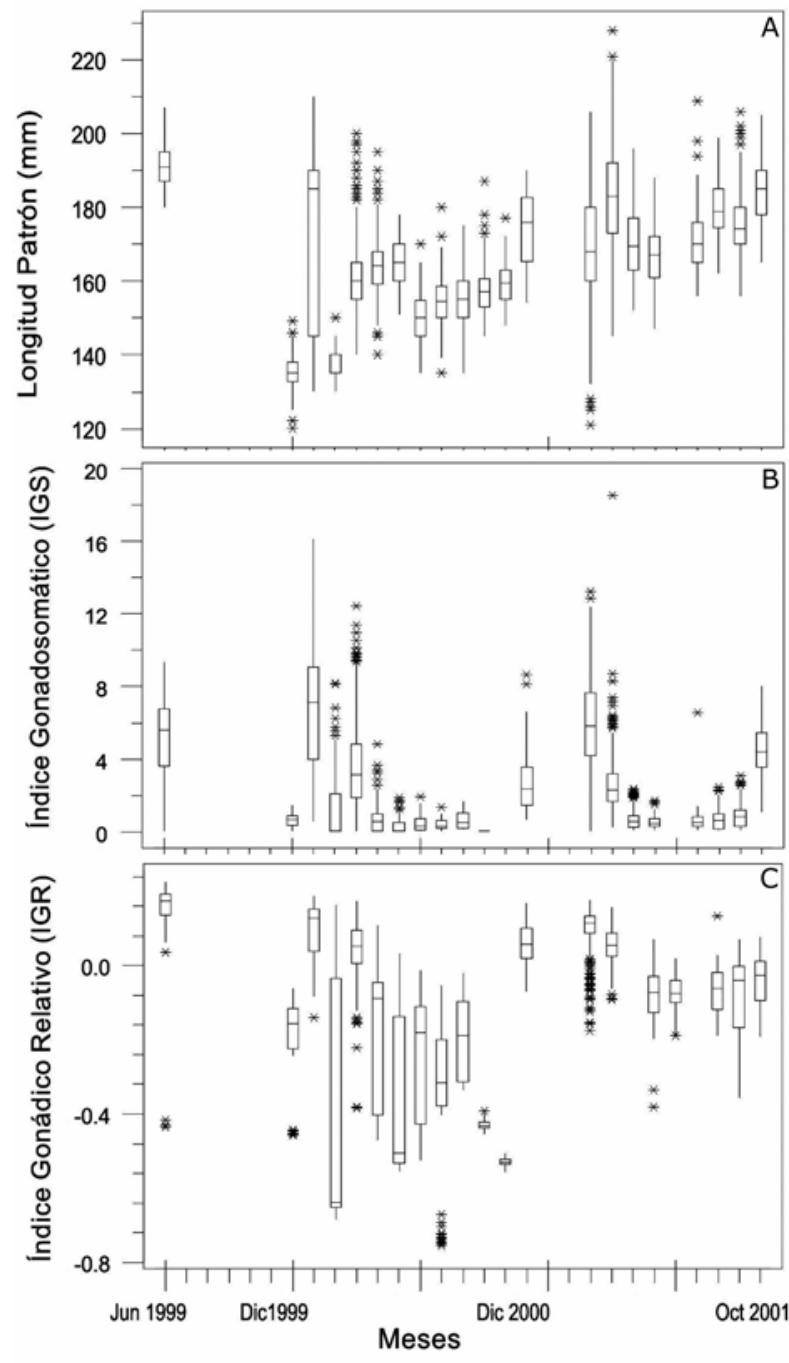

Figura 2

(a) Distribución mensual de tallas de $S$. sagax en la temporada reproductiva 1999-2000. (b) Variación del índice gonadosomático (GSI). Se indica la media y el intervalo ( $X \pm \sigma$ ). (c) Variación del índice gonádico relativo (GRI). Se indica la media y el intervalo ( $\bar{X} \pm \sigma$ ), el índice fue normalizado con la relación mensual talla - peso

(a) S. sagax monthly size distribution from reproductive season 1999 - 2000. (b) Gonadosomatic index (GSI). Variation range ( $\bar{X} \pm \sigma$ ). (c) Gonadosomatic relative index (GRI). Variation range ( $\bar{X} \pm \sigma$ ), index was normalized by monthly size - weight relationship donde la media fue de $160 \mathrm{~mm}$ (Fig. 2). El IGS en enero alcanzó una media de 7.0, declinó a 2,0 en febrero y se recuperó ligeramente en marzo a 3,0. La variación del IGR sigue el mismo patrón descrito para el IGS; aunque en este caso se observan amplios intervalos de variación relacionada con los grupos de talla presentes cada mes. Si se comparan los valores con el de junio de 1999, un mes fuera de la temporada de puesta, se observa que los valores medios del IGS y del IGR fueron bajos. Es decir, el máximo de puesta de invierno fue atenuado en la temporada reproductiva de 1999-2000 (Figs. 2b y 2c). En las figuras anteriores se incluyen los valores registrados para los distintos índices de la temporada 2000 y 2001, para fines comparativos, la cual transcurrió sin la incidencia de atresia hasta el final de la temporada reproductiva.

A partir de abril de 2000, tanto el GRI como el GSI se mantuvieron bajos con pequeñas variaciones debido a la inactividad gamética. Los índices de peso presentaron amplios intervalos de variación alrededor de la media mensual, durante la madurez sexual y el desove (Figs. 2b y 2c).

El factor de condición (FC) en enero fue de 0.01 y se incrementó a 2,3 en febrero; entre marzo y abril se mantuvo con poca variación en 0,02 ; en mayo llegó a 1.9; para junio fue de $0,7 \mathrm{y}$ el mayor incremento se dio fuera de la temporada de puesta, en julio, con 18,8. La variación de CF se muestra en la Fig. 3a, y se compara con la serie temporal 1980 - 1990 (Torres-Villegas et al. 1995). A partir de esta comparación se observa que durante la temporada de madurez 1999 - 2000, el FC fue menor de 3,0 en el pico de puesta.

El análisis histológico de los ovarios indica que la temporada de puesta 1999 - 2000 se inició en enero de 2000 y concluyó en marzo 2000. En diciembre de 1999 sólo se encontraron sardinas juveniles y no se identificaron hembras activas. En enero, la frecuencia de hembras activas alcanzó el 62\%, de las cuales el 9\% mostró atresia $\alpha$ y $\beta$ sin vitelo y el 1\% estaba en estado de alveolo cortical. En febrero, las hembras activas declinaron al $26 \%$ y la atresia $\alpha$ y $\beta$ sin vitelo bajó al $6 \%$. La frecuencia de hembras activas alcanzó el $4 \%$ en marzo, de las cuales el 13\% mostró atresia en oocitos perinucleolares y el 18\% estaba en estadios con vitelo. La atresia mayor se registró en el 90\% de las hembras, lo que indicó el final de la temporada de puesta. En abril, la actividad reproductiva había terminado por 


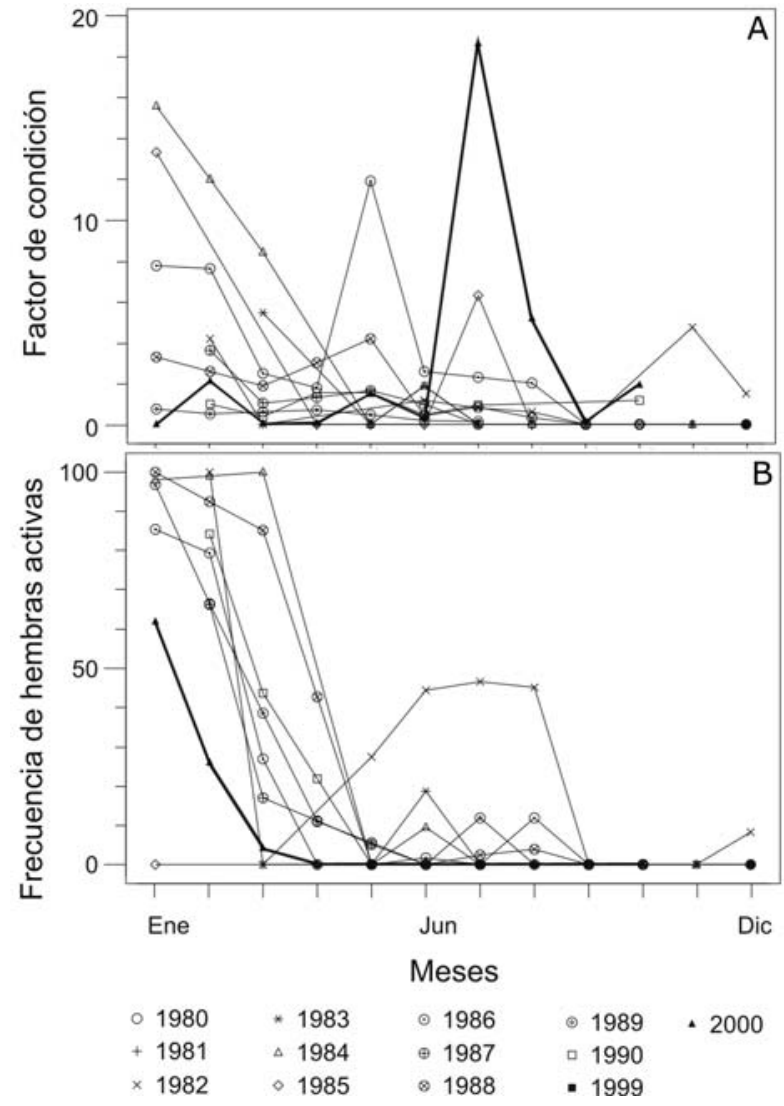

Figura 3

(a) Comparación de la frecuencia de hembras activas de S. sagax para la temporada de reproducción 1999-2000 y la serie temporal 1980-2000; (b) comparación de las series del factor de condición para los mismos períodos

(a) Comparison of 1980-1990 and 1999-2000 season time series of active female frequency for $S$. sagax, (b) time series comparison of the condition factor in the same periods

completo, con $100 \%$ de atresia mayor (Tabla 2). La frecuencia de hembras activas obtenida en este trabajo se compara con la serie temporal entre 1980 - 1990 de la población sexualmente activa para la temporada 1999 - 2000. Por otro lado, se registró la reabsorción de oocitos con cantidades importantes de vitelo, atresias $\alpha$ y $\beta$ con vitelo (ver Figs. $4 \mathrm{a}-4 \mathrm{~d}$ ), así como en oocitos perinucleolares (Figs. 4e - 4h).

En el periodo de estudio se registraron solo siete sardinas con folículos postovulatorios: tres en febrero (6\%) y cuatro en marzo (1\%). Estos resultados indican que pocas hembras alcanzaron el desove en esta temporada. Debido a la baja frecuencia de estadios postovulatorios no fue posible estimar la frecuencia de hembras desovantes.

\section{Discusión}

La temporada de puesta de $S$. sagax ha sido documentada por Torres-Villegas et al. (1995) en una serie temporal de la frecuencia de hembras activas registrada mensualmente durante 10 años en Bahía Magdalena. Esta serie muestra que la sardina monterrey desova en el área de estudio con un máximo entre diciembre y abril. En este periodo la frecuencia de hembras activas varía entre el 90 y 100\%. Se observó un segundo máximo de hembras activas entre junio y julio, fue un evento irregular, donde las hembras activas alcanzaron entre el 15 y 50\%. Dependiendo de la presencia de este segundo máximo, la temporada de desove de la sardina monterrey varía entre 4 y 8 meses. En esta serie temporal de 10 años se han registrado algunos períodos con actividad reproductiva desminuida; sin embargo, no se ha registrado la reabsorción masiva de los oocitos en el ovario.

Los resultados del seguimiento de la reproducción de la sardina monterrey en la temporada 1999 - 2000 indica que el desove se redujo a dos meses en el invierno, y el máximo se encontró cerca del 60\% del valor normalmente registrado para este máximo. De la misma forma, la incidencia de hembras maduras se presentó irregularmente retrasada, con indicadores de reabsorción de los oocitos de forma prematura en la temporada.

Por otro lado, la reabsorción de oocitos intraováricos o atresia, es un proceso normal. En algunas especies de peces pelágicos la frecuencia de atresia es normalmente alta, como en Trachurus trachurus donde es cercana al 20\% (Priede 1994). También se han registrado altas tasas de atresia en Scomber japonicus (Torres-Villegas datos no publicados). Las frecuencias de atresia cercanas al $2 \%$ durante la temporada de puesta de la sardina monterrey se consideran como normales (Torres-Villegas 1997). Cuando la atresia alcanza el 6\%, indica el final de la temporada de puesta. Comparando estos valores con los registros de la temporada 1999 - 2000, se registró atresia por encima del valor del fin del desove, justo al inicio, de manera prematura. 
Tabla 2

Resultados del análisis histológico de los ovarios de $S$. sagax de Bahía Magdalena durante el máximo reproductivo 1999-2000

Histological analysis results of $S$. sagax ovaries in the reproductive peak 1999-2000 in Bahia Magdalena

\begin{tabular}{|c|c|c|c|c|c|c|}
\hline Categoría histológica & Ene 2000 & Ene (\%) & Feb 2000 & Feb (\%) & Mar 2000 & $\operatorname{Mar}(\%)$ \\
\hline Hembras procesadas & 92 & & 53 & & 395 & \\
\hline Hembras no activas & 35 & 38 & 36 & 68 & 20 & 5 \\
\hline Hembras activas no desovantes & 57 & 62 & 14 & 26 & 16 & 4 \\
\hline \multicolumn{7}{|c|}{ Hembras activas desovantes } \\
\hline $\mathrm{POF}(0)$ & 0 & 0,00 & 0 & 0 & 0 & 0,00 \\
\hline POF(1) & 0 & 0,00 & 0 & 0 & 0 & 0,00 \\
\hline POF(2) & 0 & 0,00 & 3 & 6 & 0 & 0,00 \\
\hline $\mathrm{POF}(3)$ & 0 & 0,00 & 0 & 0 & 4 & 1 \\
\hline Atresia mayor & 0 & 0,00 & 0 & 0 & 355 & 90 \\
\hline Total & 92 & 100 & 53 & 100 & 395 & 100 \\
\hline \multicolumn{7}{|c|}{ Atresia en oocitos inmaduros } \\
\hline$\alpha$-atresia en oocitos perinucleolares & 0 & 0,00 & 0 & 0 & 3 & 0,8 \\
\hline$\alpha$-atresia sin vitelo & 0 & 0,00 & 0 & 0 & 8 & 2 \\
\hline$\alpha$-atresia con vitelo $<50 \%$ & 0 & 0,00 & 0 & 0 & 9 & 3 \\
\hline \multicolumn{7}{|c|}{ Atresia en oocitos con vitelo } \\
\hline$\alpha$-atresia con vitelo $>50 \%$ & 8 & 9 & 3 & 6 & 43 & 11 \\
\hline$\beta$-atresia sin vitelo & 0 & 0,00 & 0 & 0 & 27 & 7 \\
\hline$\beta$-atresia con vitelo & 1 & 1 & 0 & 0 & 13 & 3 \\
\hline \multicolumn{7}{|c|}{ Estados viejos de atresia } \\
\hline$\gamma$-atresia & 0 & 0,00 & 0 & 0 & 0 & 0,00 \\
\hline$\delta$-atresia & 0 & 0,00 & 0 & 0 & 0 & 0,00 \\
\hline
\end{tabular}

POF $=$ folículos postovulatorios

Establecer las causas de la interrupción de la reproducción es difícil debido a que la atresia en peces marinos está pobremente documentada, y ha sido descrita principalmente para especies dulceacuícolas cultivadas y en especies anádromas (Rideoult et al. 2000). La incidencia diferencial de atresia en oocitos sin vitelo está probablemente relacionada con la condición de los peces pequeños presentes en el área al inicio de la temporada. Hacia el final de la temporada, las sardinas adultas con atresia en oocitos con vitelo pudieran relacionarse con la condición o con un cambio ambiental que causó la interrupción de la puesta. Fedorov (1971) investigó el estado reproductivo de
Reinhardtius hippoglossoides y encontró que la reproducción no es un proceso exactamente anual, puesto que registró la degeneración de las gónadas en algunos años. Aparentemente esta especie puede pasar por alto una temporada de puesta; este autor indica una posible relación con decrementos en la temperatura. Los datos de temperatura de Bahía Magdalena (Feldman 2007), en la temporada de puesta 1999-2000, indican una baja en la temperatura. Esta coincidencia es difícil evaluarla como una relación causa - efecto, puesto que a pesar de que está documentado en algunas especies, no hay datos de la sardina monterrey. 

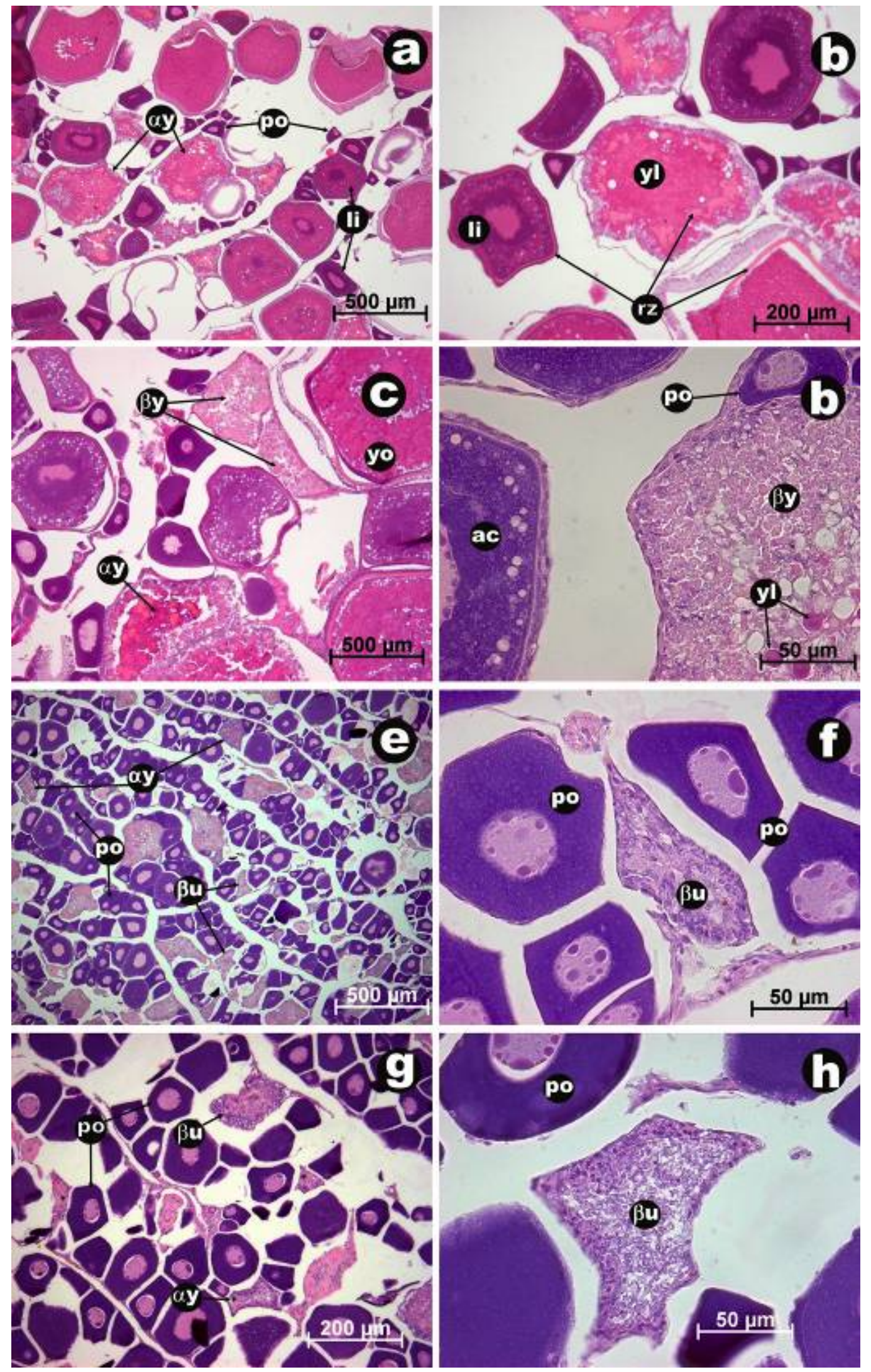


\title{
Figura 4
}

Microfotografías que muestran la atresia mayor en S. sagax. Tinción: Haematoxilina Eosina. a, Atresia mayor en un ovario maduro; b, Detalle de atresia en oocito con vitelo; $c$, Atresia mayor en ovario maduro, la figura muestra atresia alfa y beta con vitelo; d, Corporae atreticum, detalle de atresia beta con vitelo; e, Atresia mayor en un ovario inmaduro; f, Atresia beta si vitelo; g, Vista general de ovario inmaduro con atresia alfa sin vitelo y atresia beta con vitelo; $h$, Detalle de atresia beta sin vitelo

\begin{abstract}
$\alpha y=$ atresia alfa con vitelo, $\mathbf{p o}=$ oocito perinucleolar, $\mathrm{li}=$ oocito con inclusiones lipídicas, $\mathrm{yl}=$ gota de vitelo, $\mathrm{hf}=$ capas
foliculares hipertróficas, $\mathrm{rz}=$ zona radiata; $\beta \mathrm{y}=$ atresia beta con vitelo, $\mathrm{yo}=0$ ocito con vitelo; ac $=00$ ito con alvéolos corticales; $\alpha u=$ atresia alfa sin vitelo, $\beta u=$ atresia beta sin vitelo; $r z f=$ fragmentos de la zona radiada
\end{abstract}

Micrographs showing features of atresia major in S. sagax. Stain: Haematoxylin Eosin.

a, Atresia major in mature ovary,; b, Atresia on yolked oocyte (detail); c, Atresia major in mature ovary, figure shows alfa and beta yolked atresia.; d, Corporae atreticum, detail showing yolked beta atresia; e, Atresia major on immature ovary.; f. Unyolked beta atresia.; g, Immature ovary at low scale with unyolked alfa atresia and yolked beta atresia; h, Atresia on immature oocyte (detail),

Unyolked beta atresia.

$\alpha y=$ yolked alfa atresia, po = perinucleolar oocyte, li = oocyte with lipidic inclusions, $y \mathrm{l}=$ yolk drop, hf = hipertrophic follicular layers, $\mathrm{rz}=$ radiate zone, $\beta \mathrm{y}=$ yolked beta atresia, yo = yolked oocyte, ac = oocyte with cortical alveoli, $\alpha \mathrm{u}=$ unyolked alfa atresia, $\beta \mathrm{u}=$ unyolked beta atresia, $\mathrm{rzf}=$ fragments of the radiata zone

Se ha documentado también la relación entre la nutrición y la reproducción en experimentos con Salmo trutta en cautiverio (Bagenal 1969), en Gasterosteus aculeatus (Wooton 1973), en Melanogrammus aeglefinus (Hislop et al. 1978), y en Gadus morhua (Kjesbu et al. 1991). Estos autores describen que la inanición causa la suspensión del desove y reduce la tasa de producción de oocitos. Burton \& Idler (1984) asociaron la interrupción de la maduración del oocito con la condición de las hembras de Pseudopleuronectes americanus. El hecho de que el factor de condición estuviera por debajo de lo habitual sugiere un efecto ambiental de mayor plazo, puesto que la atresia mayor se observó en oocitos inmaduros así como en oocitos con vitelo. Estos registros sugieren la posibilidad de un doble efecto, por un lado la atresia mayor en oocitos maduros, con vitelo, se asocia con cambios repentinos de temperatura. Por otro lado, la reabsorción de oocitos inmaduros se ha descrito en condiciones prolongadas de inanición. Una tercera posibilidad es que se haya presentado una mezcla de ambos procesos. Con los datos disponibles de la incidencia de atresia en $S$. sagax no es posible establecer una relación causa - efecto.

La incidencia prematura de atresia mayor y una interrupción de la puesta en Gadus morhua se registró en la temporada 1999 - 2000 como en otros años en el Atlántico Norte (Messiatzeva 1932, Shirokova 1969, Burton et al. 1997). En G. morhua, la atresia ocurrió en la población adulta al inicio de la temporada reproductiva, en oocitos en estado de alveolo cortical, produciéndose posteriormente la interrupción del desove. En este caso hubo una relación directa entre el factor de condición y la falla reproductiva (Rideoult et al. 2000). En la sardina también se presenta esta relación directa con el factor de condición.

Cualquiera que sea la causa de la reabsorción del ovario, se trata de un proceso de muerte celular programada o apoptosis (Wyllie et al. 1980, Nagahama et al. 1995), y se encuentra íntimamente regulada por el ciclo hormonal. En estos términos se sugiere la influencia del ambiente en la fisiología de los peces, así como en las tasas de producción de alimento y, en general, en sus condiciones.

Picquelle \& Stauffer (1985) definen la producción de huevos como una observación instantánea; sin embargo, sobre una base anual, la producción de huevos depende de la producción per capita y de las condiciones de la población desovante. Entonces, los parámetros que definen la producción de huevos son la frecuencia de hembras participantes en la puesta y del tiempo en que este grupo se encuentra disponible para la reproducción. La frecuencia de hembras activas es un indicador del tamaño de la población disponible para la puesta; cuando esta frecuencia es notablemente baja, la fracción desovante es aún más pequeña. Infortunadamente la reducción en la fecundidad parcial no ha sido investigada para la sardina monterrey. 
Si bien se reconoce que el ambiente juega un papel determinante en los cambios en la abundancia de especies de peces pelágicos menores, se conoce poco sobre estos mecanismos. Es posible que la reabsorción de los oocitos intraováricos corresponda a la interrelación de varios parámetros ambientales, lo que pudiera ser uno de los mecanismos para explicar el cambio en abundancia. Es necesario responder cuestiones sobre la reabsorción; se requiere saber si puede ser un proceso generalizado en la población, además de establecer su relación con las variaciones en la tasa de reclutamiento. La principal limitante para estos análisis consiste en obtener observaciones de la fisiología de la sardina en las poblaciones silvestres, apoyadas en experimentación en laboratorio. Sin embargo, las observaciones en condiciones controladas sólo pueden servir como indicadores de procesos que se llevan a cabo en el medio silvestre.

\section{Agradecimientos}

Los autores agradecen a los productores de sardina de Baja California Sur, al CONACyT y al Gobierno del Estado de Baja California, así como al IPN por el apoyo financiero al proyecto "Desarrollo de indicadores ambientales para reducir el consumo de combustible en las operaciones de pesca de sardina en Baja California” (FOMIX: BC-2004-C01-017; SIP20070460). También se agradecen los comentarios y sugerencias de los evaluadores anónimos de este trabajo.

\section{Literatura citada}

Alarcón VH, SR Goldberg \& J Alheit. 1984. Histología de los folículos post-ovulatorios de la sardina (Sardinops sagax) del Perú. Boletín del Instituto del Mar, Perú 8: 514.

Bagenal TB. 1969. The relationship between food supply and fecundity in brown trout Salmo trutta L. Journal of Fish Biology 1: 167-182.

Beamish RJ, WL Lockhart, JC Vanloon \& HH Harvey. 1975. Long-term acidification of a lake and resulting effects on fishes. Ambio 4: 98-102.

Bell JD, JM Lyle, CM Bulman, KJ Graham GM Newton \& DC Smith. 1992. Spatial variation in reproduction and occurrence of non-reproductive adults in orange roughy, Hoplostethus atlanticus Collett (Trachichthydae), from south-eastern Australia. Journal of Fish Biology 40: 107122.
Burton MP \& DR Idler. 1984. The reproductive cycle in winter flounder, Pseudopleuronectes americanus (Walbaum). Canadian Journal of Zoology 62: 2563-2567.

Burton MPM, RM Penney \& S Biddiscombe. 1997. Time course of gametogenesis in Northwest Atlantic cod (Gadus morhua). Canadian Journal of Fisheries and Aquatic Science 54 (Suppl. 1): 122-131.

Byskov AG. 1978. Folicular atresia. En: Jones RE (ed). The vertebrate ovary, pp. 533-562. Plenum Press, Nueva York.

Claramunt G \& R Roa. 2001. Indirect approach to estimate spawning fraction as applied to Sardinops sagax from northern Chile. Scientia Marina 65: 87-94.

Erickson DL, JE Hightower \& D Grossman. 1985. The relative gonadal index: an alternative index for quantification of reproductive condition. Comparative Biochemistry and Physiology 81A: 117-120.

Fedorov KY. 1971. The state of the gonads of the Barents Sea Greenland halibut (Reinhardtius hippoglossoides (Walbaum)) in connection with failure to spawn. Journal of Ichthyology 11: 673-682.

Feldman GC. 2007. OceanColor Web. [en línea] NASA Ocean Color Program Washington D. C. USA. 2007 $<$ http://oceancolor.gsfc.nasa.gov/> [Consultado 9 de abril 2007].

Grier HJ. 2000. The germinal epithelium: Its dual role in establishing male reproductive classes and understanding the basis for indeterminate egg production in female fishes. Proceedings of the fifty-third Annual of the Gulf and Caribbean. Fisheries Institute 53: 537-552.

Hislop JRG, AP Robb \& JA Gauld. 1978. Observation on effects of feeding level on growth and reproduction in haddock, Melanogrammus aeglefinus (L.) in captivity. Journal of Fish Biology 13: 85-98.

Hunter JR \& BJ Macewicz. 1980. Sexual maturity, batch fecundity, spawning frequency and temporal pattern of spawning for the northern anchovy, Engraulis mordax, during the 1979 spawning season. California Cooperative Oceanic Fisheries Investigation Report 21: 139-149.

Hunter JR \& BJ Macewicz. 1985. Rates of atresia in the ovary of captive and wild northern anchovy, Engraulis mordax. Fishery Bulletin 83: 119-135.

Hunter JR \& BJ Macewicz. 2003. Improving the accuracy and precision of reproductive information used in fisheries. En: Kjesbu OS, JR Hunter \& PR Witthames (eds). Modern approaches to assess maturity and fecundity of warm and cold water fish and squids, pp. 5768. Institute of Marine Research, The Research Council of Norway, Bergen. 
Hunter JR, NCH Lo \& RJH Leong. 1985. Batch fecundity in multiple spawning fishes. NOAA Technical Report NMFS 36: 67-77.

Hunter JR, BJ Macewicz, NCH Lo \& CA Kimbrell. 1992. Fecundity spawning, and maturity of females dover sole Microstomus pacificus with an evaluation of assumption and precision. Fishery Bulletin 90: 101-128.

Kjesbu OS, J Klungsøyr, H Kryvi, PR Witthames \& M GreerWalker. 1991. Fecundity, atresia and egg size of captive Atlantic cod (Gadus morhua) in relation to proximate body composition. Canadian Journal of Fisheries and Aquatic Science 48: 2333-2343.

Lam TJ, Y Nagahama, K Chan \& WS Hoar. 1978. Overripe eggs and post-ovulatory corpora lutea in the threespine stickleback (Gasterosteus aculeatur) L., form trachurus. Canadian Journal of Zoology 56: 2029-2036.

Lambert JGD. 1970. The ovary of the guppy, Poecilia reticulata. The atretic follicle, a corpus atreticum or a corpus luteum pracovulationis. Zeitschrift für Zellforschung 107: 51-67.

Livingstone ME, M Vignaux \& KA Schofield. 1997. Estimating the annual proportion of nonspawning adults in New Zealand hoki, Macruronus novaezelandiae. Fishery Bulletin 95: 99-113.

Lowe J. 1997. Histotechnology technical methods. [on line] En: University of Nottingham Medical School Division of Histopathology. Notthingham England. May $6^{\text {th }} \cdot 1997$ <http://www.nottingham.ac.uk/pathology/default.html> [Consultado: 10 noviembre 2001].

Macer CT. 1974. The reproductive biology of the horse mackerel Trachurus trachurus (L.) in the North Sea and English Channel. Journal of Fish Biology 6: 415-438.

Maddock MD \& MPM Burton. 1994. Some effects of starvation on the lipid and skeletal muscle layers of the winter flounder, Pleuronectes americanus. Canadian Journal of Zoology 72: 1672-1679.

McFarland GA \& WG Franzin. 1978. Elevated heavy metals: a stress on a population of white sucker (Catostomus commersoni) in Hamell Lake, Saskatchewan. Journal of Fisheries Research Board of Canada 35: 963970.

Martoja R \& M Martoja-Pierson. 1970. Técnicas de histología animal, 350 pp. Toray-Masson, Barcelona.

Messiatzeva E. 1932. Chief results of the fishery research in the Barents Sea in 1930 by the GOIN (State Oceanographical Institute of USSR). Rapports et Procèsverbaux des Réunions Conseil Permanent pour l'Exploration de la Mer 81 (Appendix 3) : 141-151.

Nagahama Y, M Yoshikumi, M Yamashita, T Tokumoto \& Y Katsu. 1995. Regulation of oocyte growth and maturation in fish. Current Topics in Developmental Biology 30: 103-145.

Ochoa-Báez RI. 1998. Estacionalidad reproductiva y producción ovárica de la anchoa europea, Engraulis encrasicolus (Clupeidae: Engraulidae) en el mar Catalán. Tesis Doctoral, Universidad Politécnica de Cataluña, Barcelona, 287 pp.

Picquelle S \& G Satuffer. 1985. Parameter estimation for an egg production method of Northern anchovy biomass assessment. NOAA Technical Report NMFS 36: 7-15.

Priede IG. (Co-ordinator). 1994. Spawning biology, distribution and abundance of mackerel, Scomber scombrus and horse mackerel, Trachurus trachurus in the North East Atlantic. Final Report submitted to the Directorate-General of Fisheries (DG XIV) of the Commission of the European Communities. [Project number: MA 2436 April 1994].

Rideout RM, MPM Burton \& GA Rose. 2000. Observations on mass atresia and skipped spawning in northern Atlantic cod, from Smith Sound, Newfoundland. Journal of Fish Biology 57: 1429-1440.

Rijnsdrop AP. 1990. The mechanism of energy allocation over production and somatic growth in female North Sea plaice, Pleuronectes platessa L. Netherlands Journal of Sea Research 25: 279-290.

Scott DP. 1962. Effect of food quantity on fecundity of rainbow trout Salmo gairnieri. Journal of the Fisheries Board of Canada 19: 715-731.

Shirokova MY. 1969. The sexual maturation rate of the generations of Baltic cod taken from 1961-1963. Trudy Atlanticheskii Naucho-Issledovatel'skii Institut Rybnogo Khoziaistva i Okeanografii 21: 37-44 (en ruso).

Swingle HS. 1956. A repressive factor controlling reproduction in fishes. Proceedings of Pacific Science Congress 8: 865871.

Tesch FW. 1971. Age and growth. En: Ricker WE (ed) Methods for Assessment of Fish Production in Fresh Waters, pp. 98-130. International Biological Programme. Blackwell Scientific Publications, Oxford and Edimburg.

Torres-Villegas JR. 1986. Evaluación de Sardinops sagax por el método de producción de huevos, en Bahía Magdalena, B.C.S., México. Tesis de Maestría en Ciencias, Instituto Politécnico Nacional, CICIMAR-IPN, La Paz, Baja California Sur, 116 pp.

Torres-Villegas JR. 1997. La reproducción de la sardina monterrey Sardinops caeruleus (Girard, 1854) en el Noroeste de México y su relación con el ambiente. Tesis Doctoral, Universidad Politécnica de Cataluña, Barcelona, 338 pp. 
Torres-Villegas JR, G García, A Levy \& RI Ochoa-Báez. 1985. Madurez sexual, peso promedio, proporción de sexos y frecuencia de desovantes de Sardinops sagax en el Golfo de California para noviembre de 1984. CIBCASIO Transactions 10: 536-549.

Torres-Villegas JR, RI Ochoa-Báez, L PerezgomómezÁlvarez \& G García-Melgar. 1995. Comparison of seasonal variability in reproduction of Pacific sardine (Sardinops sagax) from Baja California Sur, Mexico, in the years 1982-1992. Scientia Marina 59: 255-264.

Torres-Villegas JR, RI Ochoa-Báez, G García Melgar, L Perezgómez \& EO Rodríguez-Class. 2000. La madurez final del folículo de la sardina monterrey (Sardinops caeruleus) y las estimaciones de la fecundidad parcial. VII Congreso Nacional de Ictiología, México: 86-87.
Trippel EA \& HH Harvey. 1990. Ovarian atresia and sex ratio inbalance in white sucker, Catostomus commersoni. Journal of Fish Biology 36: 231-239.

Wallace RA \& K Selman. 1981. Cellular and dynamic aspects of oocyte growth in teleosts. American Zoologist 21: $325-343$.

Wooton RJ. 1973. The effect of food ration on egg production in the female three-spined stickback, Gasterosteus aculeatus L. Journal of Fish Biology 5: 8996.

Wyllie AH, JFR Kerr \& AR Currie. 1980. Cell Death: The significance of apoptosis. International Review of Cytology 68: 251-306.

Recibido el 22 de mayo de 2007 y aceptado el 31 de agosto de 2007 